\title{
Low-dose MDCT: evaluation of the impact of systematic tube current reduction and sparse sampling on quantitative paraspinal muscle assessment
}

\author{
Egon Burian ${ }^{1,2}$, Nico Sollmann ${ }^{1}$, Kai Mei ${ }^{3}$, Michael Dieckmeyer ${ }^{1}$, Daniela Juncker ${ }^{2}$, Maximilian Löffler ${ }^{1}$, \\ Tobias Greve ${ }^{1,4}$, Claus Zimmer ${ }^{1}$, Jan S. Kirschke ${ }^{1}$, Thomas Baum ${ }^{1}$, Peter B. Noël ${ }^{3}$ \\ ${ }^{1}$ Department of Diagnostic and Interventional Neuroradiology, Klinikum rechts der Isar, School of Medicine, Technical University of Munich, \\ Munich, Germany; ${ }^{2}$ Department of Diagnostic and Interventional Radiology, Klinikum rechts der Isar, School of Medicine, Technical University \\ of Munich, Munich, Germany; ${ }^{3}$ Department of Radiology, Perelman School of Medicine, University of Pennsylvania, Philadelphia, PA, USA; \\ ${ }^{4}$ Department of Neurosurgery, Klinikum der Universität München, Ludwig-Maximilians-Universität, Munich, Germany
}

Correspondence to: Egon Burian, MD, DMD. Department of Diagnostic and Interventional Neuroradiology, Klinikum rechts der Isar, Technical University of Munich, Ismaninger Str. 22, 81675 Munich, Germany. Email: egon.burian@tum.de.

Background: Wasting disease entities like cachexia or sarcopenia are associated with a decreasing muscle mass and changing muscle composition. For valid and reliable disease detection and monitoring diagnostic techniques offering quantitative musculature assessment are needed. Multi-detector computed tomography (MDCT) is a broadly available imaging modality allowing for muscle composition analysis. A major disadvantage of using MDCT for muscle composition assessment is the radiation exposure. In this study we evaluated the performance of different methods of radiation dose reduction for paravertebral muscle composition assessment.

Methods: MDCT scans of eighteen subjects ( 6 males, age: $71.5 \pm 15.9$ years, and 12 females, age: $71.0 \pm 8.9$ years) were retrospectively simulated as if they were acquired at $50 \%, 10 \%, 5 \%$, and $3 \%$ of the original X-ray tube current or number of projections (i.e., sparse sampling). Images were reconstructed with a statistical iterative reconstruction (SIR) algorithm. Paraspinal muscles (psoas and erector spinae muscles) at the level of L4 were segmented in the original-dose images. Segmentations were superimposed on all lowdose scans and muscle density (MD) extracted.

Results: Sparse sampling derived mean MD showed no significant changes $(\mathrm{P}=0.57$ and $\mathrm{P}=0.22)$ down to $5 \%$ of the original projections in the erector spinae and psoas muscles, respectively. All virtually reduced tube current series showed significantly different $(\mathrm{P}>0.05)$ mean MD in the psoas and erector spinae muscles as compared to the original dose except for the images of $5 \%$ of the original tube current in the erector spinae muscle.

Conclusions: Our findings demonstrated the possibility of considerable radiation dose reduction using MDCT scans for assessing the composition of the paravertebral musculature. The sparse sampling approach seems to be promising and a potentially superior technique for dose reduction as compared to tube current reduction.

Keywords: Multi-detector computed tomography (MDCT); dose reduction; muscle composition; paraspinal muscle; sarcopenia

Submitted Nov 01, 2020. Accepted for publication Feb 18, 2021.

doi: $10.21037 /$ qims-20-1220

View this article at: http://dx.doi.org/10.21037/qims-20-1220 


\section{Introduction}

Cachexia and sarcopenia are katabolic diseases which have great impact on muscle structure and composition (1-3). In course of wasting diseases the assessment and monitoring of overall muscle composition plays a pivotal role for initiation and adaption of further treatment steps ranging from nutritional supplements to adjustment of chemotherapy (3). In all diseases affecting the musculoskeletal system monitoring the composition of large or representative muscle groups is a matter of special interest to track the individual disease progression (4). In literature analogous fatty infiltration patterns of the paravertebral musculature with the thigh have been described before (5). As the paravertebral musculature seems to underlie the same katabolic patterns in muscle decay as the large muscle groups, it can be considered suitable for overall muscle status assessment. Furthermore, the psoas muscle has been identified as an important target muscle for tumor related sarcopenia/cachexia (6). Weerink et al. reported that the presence of low psoas mass prior to surgery, as an indicator for sarcopenia, and a strong predictor for the development of postoperative complications (6). In further studies the psoas muscle was used as target muscle for prediction of complications and survival in patients with gastrectomy, cystectomy, and liver surgery (7-9).

There is a plentitude of imaging methods in clinical use from dual energy X-ray absorptiometry (DXA) and multi-detector computed tomography (MDCT) to singlevoxel proton magnetic resonance spectroscopy (MRS) and chemical shift encoding-based water-fat magnetic resonance imaging (MRI) (10-15). Although, MRS and MRI enable the investigator to extract surrogate parameters of the muscle composition like the fat fraction (FF) and even for the identification of the chemical structure of fatty acids and their magnitude with high congruence to histology, they require advanced knowledge during image acquisition and are rather high priced (15). MDCT is broadly available in even smaller hospitals' allocation and used in monitoring of oncological patients, liver fat and subcutaneous-/ visceral fat assessment $(16,17)$. The number of MDCT scans and corresponding radiation dose a subject received, was shown to be directly correlated with estimated individual cancer risk (18-20). One possibility to tackle this modality specific issue is to reduce the radiation dose received by each subject undergoing an MDCT scan.

Radiation dose reduction can generally be achieved by different approaches including lowering of tube current or by using sparse sampling, which is a technique that allows for acquisition of fewer projections in MDCT scans $(21,22)$. However, the extent of tube current attenuation and decrease of projection numbers is limited with regard to preservation of scan quality $(23,24)$. Sparse sampling is a technologically advanced method in which radiation exposure is lowered as a consequence of decreased projection numbers, whereas the needed energy delivery is stable (23).

In this study the limits of virtual tube current reduction of MDCT were compared to the sparse sampling technique and the validity of the acquired images for the assessment of paraspinal muscle composition was investigated.

\section{Methods}

\section{Subjects}

Eighteen patients were included in this retrospective study (6 males, age: $71.5 \pm 15.9$ years, and 12 females, age: $71.0 \pm 8.9$ years). The included patients underwent noncontrast MDCT imaging of the spine at our department due to suspected degenerative spine diseases or trauma. Our hospital's picture archiving and communication system (PACS, IDS7; Sectra AB, Linköping, Sweden) was used for patient identification.

Identification criteria were: (I) existing MDCT scan of the lumbar vertebral spine, (II) no medical history of metabolic or muscular diseases, (III) no relevant, image quality reducing artifacts, (IV) no previous surgery with instrumentation at the spine, and (5) no presence of any implants in the field of view.

This study was approved by the local institutional review board (62/18S) and was conducted in accordance with the Declaration of Helsinki. Written and oral informed consent for future anonymized data processing was given by each participant when signing the informed consent before the CT scan.

\section{$M D C T$}

Image acquisition was performed in supine position using a 128-slice MDCT scanner (Ingenuity Core 128; Philips Healthcare, Cleveland, OH). An initial scout scan was used for planning purposes. Then a helical scan was performed with implicit tube current modulation. Scan parameters are shown in Table 1. 
Table 1 Scan parameters

\begin{tabular}{lc}
\hline Parameter & Value \\
\hline Tube voltage $(\mathrm{kV})$ & 120 \\
Rotation time $(\mathrm{s})$ & $0.78 \pm 0.163$ \\
Exposure $(\mathrm{mAs})$ & $194.5 \pm 56.8$ \\
Voxel spacing $\left(\mathrm{mm}^{3}\right)$ & $0.39 \times 0.39 \times 0.90$ \\
CTDI $(\mathrm{Gy})$ & $13.8 \pm 5.0$ \\
DLP $\left(\mathrm{mG}{ }^{*} \mathrm{~cm}\right)$ & $388.9 \pm 179.9$ \\
Slice thickness $(\mathrm{mm})$ & 0.9 \\
\hline
\end{tabular}

CTDI, CT dose index; DLP, dose-length product.

\section{Virtual tube current reduction}

Tube current reduction was performed virtually. For this purpose, a simulation algorithm based on raw imaging data was used, which allowed for generating MDCT scans with virtually lowered tube currents (25-27). The original scan with full dose without dose reduction or sparse sampling was defined as D100P100. The corresponding simulations had 50\% (D50P100), 10\% (D10P100), 5\% (D5P100), and $3 \%$ (D3P100) of the original X-ray tube current.

\section{Sparse sampling and statistical iterative reconstruction (SIR)}

Sparse sampling was simulated by reading only a reduced amount of projection angles and by deleting the remaining projections in the sonogram $(27,28)$. Sparse-sampled images were generated as if MDCT was performed with only $50 \%$ (D100P50), 10\% (D100P10), 5\% (D100P5), and 3\% (D100P3) of the original projection data.

\section{SIR}

For both virtual tube current reduction as well as sparse sampling the same in-house developed SIR algorithm that was based on ordered-subset separable paraboloidal surrogate combining a momentum accelerating approach was used $(29,30)$. In detail, a Gaussian noise model was applied and the likelihood term for SIR was computed with log-converted projection data. To enhance convergence and to further depress image noise while achieving adequate bone/soft tissue contrast, a regularization term based on a Huber penalty was applied. The calibration data served for calculating linear attenuation coefficients of resulting imaging data, which were then translated to Hounsfield units by using air and water information.

\section{Segmentation of the psoas muscle and the erector spinae and muscle density (MD) measurements}

The psoas muscle and the erector spinae muscles were segmented bilaterally at the level of L4 over a craniocaudal distance of $10 \mathrm{~mm}$ in the axial reformations of the original dose images (Figure 1). Segmentation was performed by using the free open-source software Medical Imaging Interaction Toolkit (MITK, developed by the Division of Medical and Biological Informatics, German Cancer Research Center, Heidelberg, Germany) by a radiologist with 4 years of experience. Segmentations were superimposed on all low-dose scans. MD was extracted in Hounsfield Units (HU) of each muscle in each scan. Volume weighted mean MD of the right and left psoas and erector spinae muscle was calculated, respectively. A second reader performed segmentation of all included subjects to evaluate the inter-rater reliability of the results.

\section{Signal-to-noise ratio (SNR) calculation}

The SNR from each virtually reduced and each sparse sampling series was calculated as follows: $\mathrm{SNR}=$ mean in $\mathrm{HU} /$ standard deviation in HU.

\section{Statistical analysis}

For the statistical analyses SPSS (version 22.0; IBM SPSS Statistics for Windows, Armonk, NY, USA) was used. Statistical significance was considered at $p<0.05$ (two-sided) in all conducted tests.

The Kolmogorov-Smirnov test indicated normally distributed data.

MD values derived from virtually lowered tube current and sparse sampling were compared with each other and with those derived from original dose images as gold standard by using paired t-tests. To assess the reliability of the acquired results, intraclass correlation coefficient (ICC) was calculated.

\section{Results}

Mean MD was $6.41 \pm 13.55 \mathrm{HU}$ for the erector spinae muscles derived from original dose images (D100P100). Sparse 

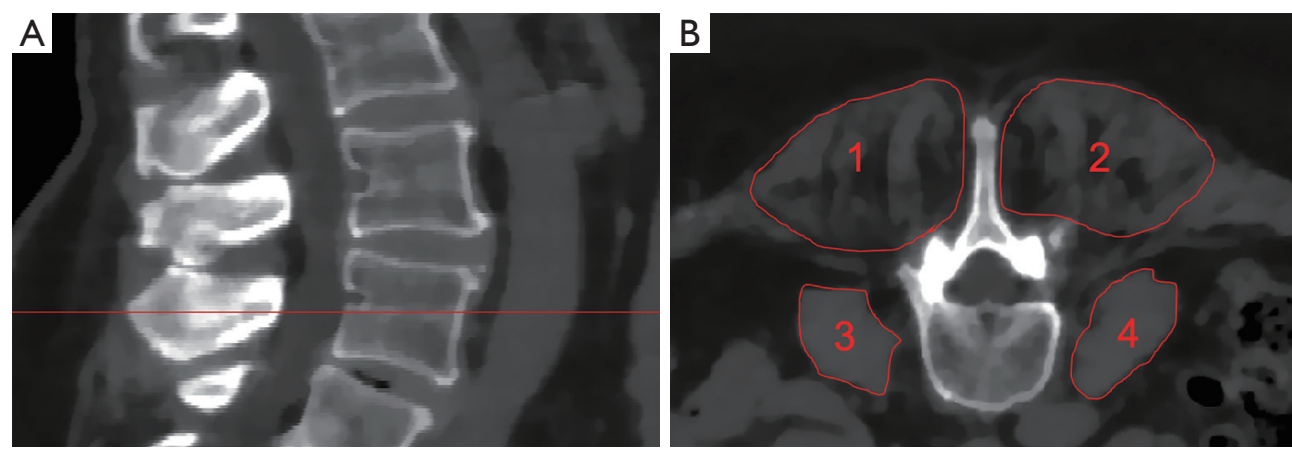

Figure 1 In this figure representative segmentations are depicted. The level L4 (A) was chosen to perform manual segmentation of the right (1) and left (2) erector spina and the right (3) and left (4) psoas muscle (B).

Table 2 Extracted Hounsfield units (HU) for each sparse sampling (D100P50, D100P10, D100P5 and D100P3) and virtual dose reduced series (D50P100, D10P100, D5P100 and D3P100) are shown and compared to the original dose (D100P100)

\begin{tabular}{|c|c|c|c|c|}
\hline Dose reduction series & Erector spinae (HU) & $\mathrm{P}$ & Psoas muscle (HU) & $\mathrm{P}$ \\
\hline D100P50 & $6.43 \pm 13.53$ & 0.73 & $39.97 \pm 6.06$ & 0.07 \\
\hline D100P10 & $6.51 \pm 13.58$ & 0.20 & $40.13 \pm 6.28$ & 0.02 \\
\hline D100P5 & $6.32 \pm 13.52$ & 0.57 & $40.10 \pm 6.53$ & 0.22 \\
\hline D50P100 & $6.66 \pm 13.49$ & $<0.01$ & $40.11 \pm 6.04$ & $<0.01$ \\
\hline D10P100 & $8.31 \pm 13.41$ & $<0.01$ & $40.86 \pm 6.22$ & 0.04 \\
\hline D5P100 & $5.07 \pm 15.32$ & 0.19 & $34.51 \pm 11.00$ & 0.03 \\
\hline D3P100 & $12.31 \pm 19.70$ & $<0.01$ & $14.00 \pm 17.83$ & $<0.01$ \\
\hline
\end{tabular}

sampling derived mean MD showed no significant changes $(\mathrm{P}=0.57)$ down to $5 \%$ of the original projections (D100P5). For the psoas muscle, mean MD amounted 39.86 $26.14 \mathrm{HU}$. No significant changes in mean $\mathrm{MD}$ of the psoas muscle were observed down to D100P5 ( $\mathrm{P}=0.22)$ (Table 2 and Figure 2). Representative images of virtually lowered tube current and sparse sampling are shown in Figure 2.

The virtual tube lowering showed no significant $(\mathrm{P}>0.05)$ changes for the mean $\mathrm{MD}$ of the erector spinae at $5 \%$ of the original dose D5P100 ( $\mathrm{P}=0.19)$ as compared to D100P100. However, mean MD derived from D50P100, D10P100 and D3P100 were significantly different as compared to D100P100 $(\mathrm{P}<0.01)$. In the psoas muscle, all virtually reduced tube current series showed significantly different mean MD as compared to the original dose.

The comparison of corresponding sparse sampling and virtually tube reduced series (D100P50/D50P100,
D100P10/D10P100, D100P5/D5P100 and D100P3/ D3P100) is shown in Table 3. The 50\% and $10 \%$ reduced series of the psoas and the $5 \%$ reduced series of the erector spinae MD did not differ significantly from each other ( $\mathrm{P}>0.05)$ (Figure 3).

The Signal-to-noise ratio (SNR) for each virtually tube reduced series is shown in Table 4. In the erector spinae the SNR did not reveal significant differences for the sparse sampling series down to 5\% (D100P5) of the original dose $(\mathrm{P}=0.08)$. A high ICC was given between the two readers in both muscle compartments $(\mathrm{ICC}=0.98, \mathrm{P}<0.01)$.

\section{Discussion}

In this study, we compared different approaches to effectively minimize radiation exposure in MDCT scans using virtual tube current reduction and sparse sampling. 


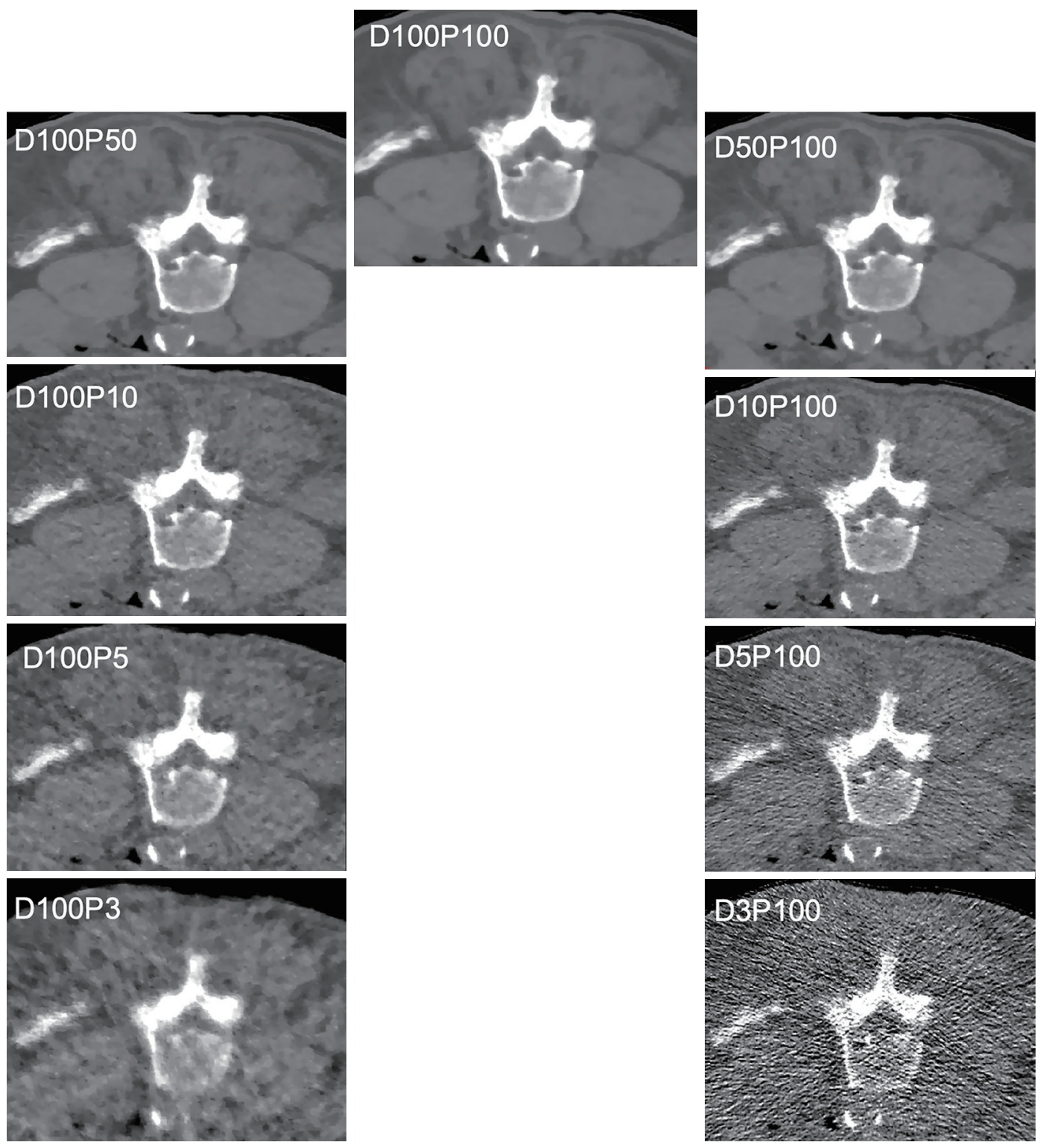

Figure 2 In this figure virtual images acquired by virtual tube current reduction and sparse sampling are shown. On the left side axial slices with 50\% (D100P50), 10\% (D100P10), 5\%(D100P5) and 3\% (D100P3) of the original projections are shown. Exemplary slices with 50\% (D50P100), 10\% (D10P100), 5\% (D5P100) and 3\% (D3P100) of the original tube current are presented on the right side.

With sparse sampling, non-significant changes in measured MD could be detected with decreased projection numbers down to $5 \%$ of the original data. In contrast, MD extracted from virtually reduced tube current images was considerably affected already at $50 \%$ of the original dose.

In clinical settings, sparse sampling could be an option in the future with regard to body composition assessment by measuring the MD of representative muscle groups with drastically minimized radiation exposure. Sollmann et al. already showed the feasibility of sparse sampling not only for BMD determination, but also in fracture diagnostic and imaging of degenerative changes of the spine $(25,26,31)$. However, available MDCT scanners are not yet capable of applying sparse sampling, because of the continuous $\mathrm{X}$-ray delivery during the scanning procedure, which is a shortcoming that might be tackled by future scanner generations.

The presented study showed not only a future perspective 
Table 3 Comparison between sparse sampling and virtual tube current reduction based HUs in the erector spinae and psoas muscles using paired $t$-tests with corresponding $\mathrm{P}$ values

\begin{tabular}{lcc}
\hline & Erector spinae & Psoas muscle \\
\hline D50P100/D100P50 & $<0.01$ & 0.76 \\
D10P100/D100P10 & $<0.01$ & 0.16 \\
D5P100/D100P5 & 0.25 & 0.02 \\
D3P100/D100P3 & $<0.01$ & $<0.01$ \\
\hline
\end{tabular}
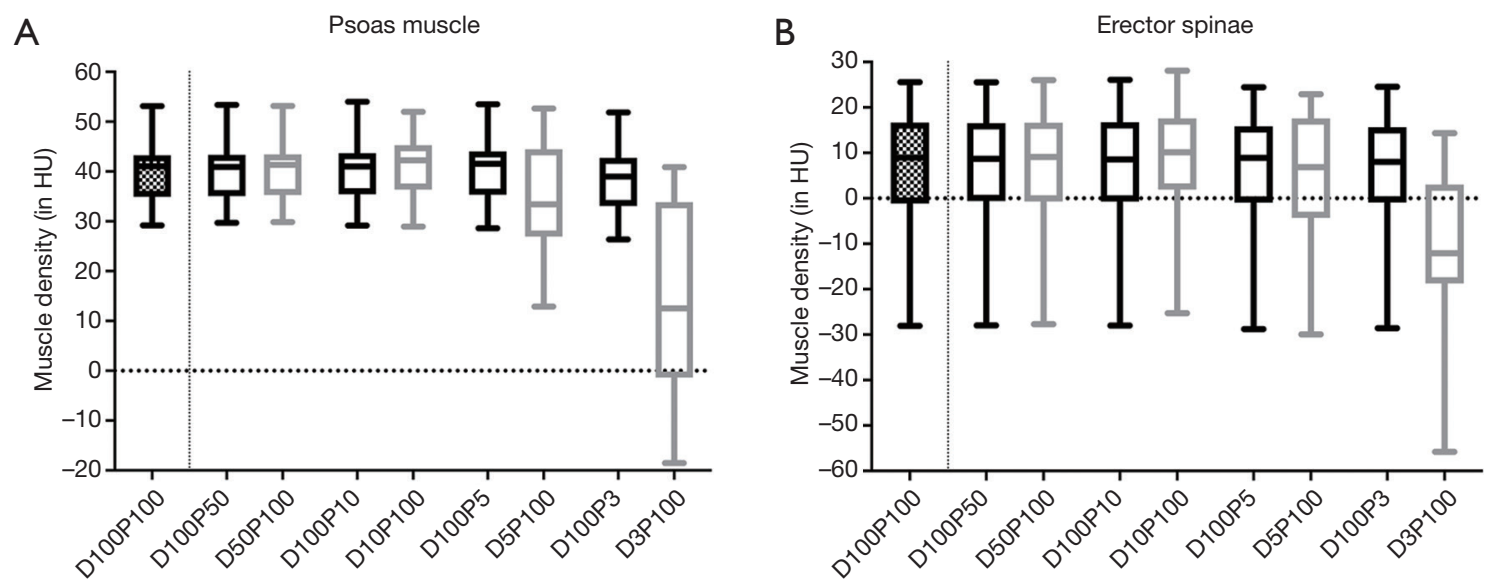

Figure 3 Muscle density measurements (MD) after virtual tube current reduction (D50P100, D10P100, D5P100 and D3P100) and sparse sampling (D100P50, D100P10, D100P10 and D100P3) are plotted for the psoas muscle (A) and the erector spinae (B). All measurements are compared to the original full dose MDCT (D100P100).

of sparse sampling, but also pointed to the possibility of applying much lower tube currents in combination with iterative image reconstruction to assess katabolic muscle changes in course of sarcopenia and cachexia. The presented findings can be directly transferred to clinical practice as a lowering of tube current can be achieved without technical effort. An aspect which should be kept in mind considering the application of tube current reduction is the increase of image noise and artifacts accompanying the scan (32). The reason for overall better results for sparse-sampled data most likely relates to the inherently different method of sparse sampling compared to tube current reduction. Specifically, while the approach of tube current reduction leads to considerable increases in image noise, that can negatively affect diagnostic use, sparse sampling implies maintained energy delivery for the individual projection image, but lowered overall radiation exposure due to the decrease in total projection numbers $(33,34)$. Consequently, preserved image quality for the individual projection can be achieved whilst circumventing the influence of electronic readout noise, leading to largely preserved structural image information.

In the past, multiple approaches for dose reduction in MDCT-based body composition imaging were performed. Amongst others, Chang et al. showed the possibility of tube current reduction up to $50 \%$ of original doses in liver imaging with a preservation of image quality and contrast-to-noise ratio and stable quantitative measures $(35,36)$. Furthermore, Yamada et al. proved the feasibility of lowering radiation doses to $30 \%$ of the original dose in imaging visceral and subcutaneous adipose tissue, which constitute the largest share of human fat deposition localizations (37). Taking the results of these groups into consideration, Elhamiasl and Nuyts described a phantombased technical approach to identifying the lowest exposure scan which still produces sufficient information for clinical applications analyzing the noise power spectrum of each dose-reduced image (38). In the proposed model presented in this study several parameters influencing image quality (like electrical noise, crosstalk between detector channels 
Table 4 The Signal to noise ratio (SNR) for each sparse sampling (D100P50, D100P10, D100P5 and D100P3) and virtual dose reduced series (D50P100, D10P100, D5P100 and D3P100) is shown and compared to the original dose (D100P100)

\begin{tabular}{|c|c|c|c|c|}
\hline Dose reduction series & Erector spinae (SNR) & $\mathrm{P}$ & Psoas muscle (SNR) & $\mathrm{P}$ \\
\hline D100P50 & $0.23 \pm 0.43$ & 0.60 & $2.04 \pm 0.81$ & 0.06 \\
\hline D100P10 & $0.21 \pm 0.39$ & 0.10 & $1.67 \pm 0.70$ & $<0.01$ \\
\hline D100P5 & $0.18 \pm 0.34$ & 0.08 & $1.35 \pm 0.59$ & $<0.01$ \\
\hline D50P100 & $0.23 \pm 0.42$ & 0.01 & $2.05 \pm 0.80$ & 0.04 \\
\hline D10P100 & $0.25 \pm 0.38$ & 0.16 & $1.64 \pm 0.69$ & $<0.01$ \\
\hline D5P100 & $0.12 \pm 0.33$ & 0.02 & $0.91 \pm 0.69$ & $<0.01$ \\
\hline D3P100 & $-0.10 \pm 0.23$ & $<0.01$ & $0.23 \pm 0.38$ & $<0.01$ \\
\hline
\end{tabular}

SNR is calculated as follows: SNR = Mean (HU)/SD (HU). SNR is displayed in arbitrary units.

and the beam hardening effect amongst others) have been taken into account to determine the lowest possible radiation dose that still produces sufficient information for proton therapy treatment planning (38).

To best of our knowledge, our presented results are the first that affirm the insights these prior studies gave into dose reduction in body composition imaging on basis of muscle density measurements. Within the limits of disease progression monitoring in entities like cachexia and sarcopenia the revealed potential but also the limits of sparse sampling based projection number reduction could be useful for clinical purposes in the future.

With that said, there are certain limitations that come along with the presented study. First, the diagnostic value of the dose or projection reduced MDCT scans with regard to other pathologies is mitigated. Like Jensen $e t$ al. showed the detection and evaluation of liver metastases is compromised in modest radiation dose reduction (39). Second, the presented cohort is rather small comprising no patients suffering from cachexia or from an oncological disease, which is a shortcoming keeping in mind the potential future application settings. Last, sparse sampling is not applicable in current clinical settings due to technical scanner properties, although it showed promising results and will pose an option for upcoming MDCT scanner generations.

\section{Conclusions}

The reported insights into virtual tube current reduction and sparse sampling based muscle density evaluation of the psoas and erector spinae muscles suggest the possibility of considerable dose reduction in MDCT scans with image quality preservation. Additionally, the results of the current study showed the potential of sparse sampling for future clinical applications in body composition imaging.

\section{Acknowledgments}

Funding: The present work was supported by the Deutsche Forschungsgemeinschaft (DFG, German Research Foundation) - Project 432290010 (to TB, PBN and JSK).

\section{Footnote}

Conflicts of Interest: All authors have completed the ICMJE uniform disclosure form (available at http://dx.doi. org/10.21037/qims-20-1220). The authors have no conflicts of interest to declare.

Ethical Statement: This study was approved by the Institutional Review Board and was conducted in accordance with the Declaration of Helsinki. Written informed consent was obtained from all study participants.

Open Access Statement: This is an Open Access article distributed in accordance with the Creative Commons Attribution-NonCommercial-NoDerivs 4.0 International License (CC BY-NC-ND 4.0), which permits the noncommercial replication and distribution of the article with the strict proviso that no changes or edits are made and the original work is properly cited (including links to both the formal publication through the relevant DOI and the license). 
See: https://creativecommons.org/licenses/by-nc-nd/4.0/.

\section{References}

1. Batsis JA, Villareal DT. Sarcopenic obesity in older adults: aetiology, epidemiology and treatment strategies. Nat Rev Endocrinol 2018;14:513-37.

2. Filippin LI, Teixeira VN, da Silva MP, Miraglia F, da Silva FS. Sarcopenia: a predictor of mortality and the need for early diagnosis and intervention. Aging Clin Exp Res 2015;27:249-54.

3. Ni J, Zhang L. Cancer Cachexia: Definition, Staging, and Emerging Treatments. Cancer Manag Res 2020;12:5597-605.

4. Spitali P, Hettne K, Tsonaka R, Charrout M, van den Bergen J, Koeks Z, Kan HE, Hooijmans MT, Roos A, Straub V, Muntoni F, Al-Khalili-Szigyarto C, KoelSimmelink MJA, Teunissen CE, Lochmüller H, Niks EH, Aartsma-Rus A. Tracking disease progression noninvasively in Duchenne and Becker muscular dystrophies. J Cachexia Sarcopenia Muscle 2018;9:715-26.

5. Burian E, Inhuber S, Schlaeger S, Dieckmeyer M, Klupp E, Franz D, Weidlich D, Sollmann N, Löffler M, Schwirtz A, Rummeny EJ, Zimmer C, Kirschke JS, Karampinos DC, Baum T. Association of thigh and paraspinal muscle composition in young adults using chemical shift encoding-based water-fat MRI. Quant Imaging Med Surg 2020;10:128-36.

6. Weerink LBM, van der Hoorn A, van Leeuwen BL, de Bock GH. Low skeletal muscle mass and postoperative morbidity in surgical oncology: a systematic review and meta-analysis. J Cachexia Sarcopenia Muscle 2020;11:636-49.

7. Cornet M, Lim C, Salloum C, Lazzati A, Compagnon P, Pascal G, Azoulay D. Prognostic value of sarcopenia in liver surgery. J Visc Surg 2015;152:297-304.

8. Yaguchi Y, Kumata Y, Horikawa M, Kiyokawa T, Iinuma H, Inaba T, Fukushima R. Clinical Significance of Area of Psoas Major Muscle on Computed Tomography after Gastrectomy in Gastric Cancer Patients. Ann Nutr Metab 2017;71:145-9.

9. Ahmadi H, Montie JE, Weizer AZ, Morgan T, Montgomery JS, Lee CT. Patient Psoas Muscle Mass as a Predictor of Complications and Survival After Radical Cystectomy. Curr Urol Rep 2015;16:79.

10. Löffler MT, Sollmann N, Mei K, Valentinitsch A, Noël PB, Kirschke JS, Baum T. X-ray-based quantitative osteoporosis imaging at the spine. Osteoporos Int 2020;31:233-50.
11. Baum T, Cordes C, Dieckmeyer M, Ruschke S, Franz D, Hauner H, Kirschke JS, Karampinos DC. MR-based assessment of body fat distribution and characteristics. Eur J Radiol 2016;85:1512-8.

12. Burian E, Syväri J, Holzapfel C, Drabsch T, Kirschke JS, Rummeny EJ, Zimmer C, Hauner H, Karampinos DC, Baum T, Franz D. Gender- and Age-Related Changes in Trunk Muscle Composition Using Chemical Shift Encoding-Based Water-Fat MRI. Nutrients 2018;10:1972.

13. Sollmann N, Dieckmeyer M, Schlaeger S, Rohrmeier A, Syvaeri J, Diefenbach MN, Weidlich D, Ruschke S, Klupp E, Franz D, Rummeny EJ, Zimmer C, Kirschke JS, Karampinos DC, Baum T. Associations Between Lumbar Vertebral Bone Marrow and Paraspinal Muscle Fat Compositions-An Investigation by Chemical Shift Encoding-Based Water-Fat MRI. Front Endocrinol (Lausanne) 2018;9:563.

14. Franz D, Weidlich D, Freitag F, Holzapfel C, Drabsch T, Baum T, Eggers H, Witte A, Rummeny EJ, Hauner $\mathrm{H}$, Karampinos DC. Association of proton density fat fraction in adipose tissue with imaging-based and anthropometric obesity markers in adults. Int $\mathrm{J}$ Obes (Lond) 2018;42:175-82.

15. Karampinos DC, Ruschke S, Dieckmeyer M, Diefenbach M, Franz D, Gersing AS, Krug R, Baum T. Quantitative MRI and spectroscopy of bone marrow. Quantitative MRI and spectroscopy of bone marrow. J Magn Reson Imaging 2018;47:332-53.

16. Graffy PM, Sandfort V, Summers RM, Pickhardt PJ. Automated Liver Fat Quantification at Nonenhanced Abdominal CT for Population-based Steatosis Assessment. Radiology 2019;293:334-42.

17. Gao Y, Wang YC, Lu CQ, Zeng C, Chang D, Ju $\mathrm{S}$. Correlations between the abdominal fat-related parameters and severity of coronary artery disease assessed by computed tomography. Quant Imaging Med Surg 2018;8:579-87.

18. Richards PJ, George J, Metelko M, Brown M. Spine computed tomography doses and cancer induction. Spine (Phila Pa 1976) 2010;35:430-3.

19. Richards PJ, George J. Diagnostic CT radiation and cancer induction. Skeletal Radiol 2010;39:421-4.

20. Smith-Bindman R, Lipson J, Marcus R, Kim KP, Mahesh M, Gould R, Berrington de González A, Miglioretti DL. Radiation dose associated with common computed tomography examinations and the associated lifetime attributable risk of cancer. Arch Intern Med 
2009;169:2078-86.

21. Omoumi P, Verdun FR, Ben Salah Y, Vande Berg BC, Lecouvet FE, Malghem J, Ott JG, Meuli R, Becce F. Lowdose multidetector computed tomography of the cervical spine: optimization of iterative reconstruction strength levels. Acta Radiol 2014;55:335-44.

22. Becce F, Ben Salah Y, Verdun FR, Vande Berg BC, Lecouvet FE, Meuli R, Omoumi P. Computed tomography of the cervical spine: comparison of image quality between a standard-dose and a low-dose protocol using filtered back-projection and iterative reconstruction. Skeletal Radiol 2013;42:937-45.

23. Abbas S, Lee T, Shin S, Lee R, Cho S. Effects of sparse sampling schemes on image quality in low-dose CT. Med Phys 2013;40:111915.

24. Willemink MJ, Noel PB. The evolution of image reconstruction for CT-from filtered back projection to artificial intelligence. Eur Radiol 2019;29:2185-95.

25. Sollmann N, Mei K, Hedderich DM, Maegerlein C, Kopp FK, Löffler MT, Zimmer C, Rummeny EJ, Kirschke JS, Baum T, Noël PB. Multi-detector CT imaging: impact of virtual tube current reduction and sparse sampling on detection of vertebral fractures. Eur Radiol 2019;29:3606-16.

26. Sollmann N, Mei K, Schwaiger BJ, Gersing AS, Kopp FK, Bippus R, Maegerlein C, Zimmer C, Rummeny EJ, Kirschke JS, Noël PB, Baum T. Effects of virtual tube current reduction and sparse sampling on MDCT-based femoral BMD measurements. Osteoporos Int 2018;29:2685-92.

27. Muenzel D, Koehler T, Brown K, Zabić S, Fingerle AA, Waldt S, Bendik E, Zahel T, Schneider A, Dobritz M, Rummeny EJ, Noël PB. Validation of a low dose simulation technique for computed tomography images. PLoS One 2014;9:e107843.

28. Zabić S, Wang Q, Morton T, Brown KM. A low dose simulation tool for CT systems with energy integrating detectors. Med Phys 2013;40:031102.

29. Rayudu NM, Anitha DP, Mei K, Zoffl F, Kopp FK, Sollmann N, Löffler MT, Kirschke JS, Noël PB, Subburaj $\mathrm{K}$, Baum T. Low-dose and sparse sampling MDCT-based femoral bone strength prediction using finite element analysis. Arch Osteoporos 2020;15:17.

30. Kim D, Ramani S, Fessler JA. Combining ordered subsets and momentum for accelerated X-ray CT image reconstruction. IEEE Trans Med Imaging 2015;34:167-78.

31. Sollmann N, Mei K, Riederer I, Probst M, Löffler MT, Kirschke JS, Noël PB, Baum T. Low-dose MDCT: evaluation of the impact of systematic tube current reduction and sparse sampling on the detection of degenerative spine diseases. Eur Radiol 2021;31:2590-600.

32. Duan X, Wang J, Leng S, Schmidt B, Allmendinger T, Grant K, Flohr T, McCollough CH. Electronic noise in CT detectors: Impact on image noise and artifacts. AJR Am J Roentgenol 2013;201:W626-32.

33. Willemink MJ, Leiner T, de Jong PA, de Heer LM, Nievelstein RA, Schilham AM, Budde RP. Iterative reconstruction techniques for computed tomography part 2: initial results in dose reduction and image quality. Eur Radiol 2013;23:1632-42.

34. Noël PB, Renger B, Fiebich M, Münzel D, Fingerle AA, Rummeny EJ, Dobritz M. Does iterative reconstruction lower CT radiation dose: evaluation of 15,000 examinations. PLoS One 2013 Nov 26;8:e81141.

35. Zhang X, Chen J, Yu N, Ren Z, Tian Q, Tian X, He T, Guo C. Improving image quality with model-based iterative reconstruction at quarter of nominal dose in upper abdominal CT. Br J Radiol 2019;92:20180137.

36. Chang W, Lee JM, Lee K, Yoon JH, Yu MH, Han JK, Choi BI. Assessment of a model-based, iterative reconstruction algorithm (MBIR) regarding image quality and dose reduction in liver computed tomography. Invest Radiol 2013;48:598-606.

37. Yamada $Y$, Jinzaki $M$, Niijima $Y$, Hashimoto $M$, Yamada M, Abe T, Kuribayashi S. CT Dose Reduction for Visceral Adipose Tissue Measurement: Effects of Model-Based and Adaptive Statistical Iterative Reconstructions and Filtered Back Projection. AJR Am J Roentgenol 2015;204:W677-83.

38. Elhamiasl M, Nuyts J. Low-dose x-ray CT simulation from an available higher-dose scan. Phys Med Biol 2020;65:135010.

39. Jensen CT, Wagner-Bartak NA, Vu LN, Liu X, Raval B, Martinez D, Wei W, Cheng Y, Samei E, Gupta S. Detection of Colorectal Hepatic Metastases Is Superior at Standard Radiation Dose CT versus Reduced Dose CT. Radiology 2019;290:400-9.

Cite this article as: Burian E, Sollmann N, Mei K, Dieckmeyer M, Juncker D, Löffler M, Greve T, Zimmer C, Kirschke JS, Baum T, Noël PB. Low-dose MDCT: evaluation of the impact of systematic tube current reduction and sparse sampling on quantitative paraspinal muscle assessment. Quant Imaging Med Surg 2021;11(7):3042-3050. doi: 10.21037/qims-201220 